\title{
Comparison of two ophthalmic viscosurgical devices used in Phaecoemulsification
}

\author{
Azzam A. Ahmed*, Muna M. Ahmed** \\ * Department of Surgery; **Department of Community Medicine, College of Medicine, University of Mosul.
}

(Ann. Coll. Med. Mosul 2012; 38 (2): 16-22).

Received: $31^{\text {st }}$ Oct. 2010; Accepted: $25^{\text {th }}$ Dec. 2011.

\begin{abstract}
Objective: To compare the effect of two ophthalmic viscosurgical devices OVDs (DisCoVisc, Alcon, Fort Worth, Taxes), and (Appavisc, Appassamy ocular devices PVT. LTD.), that currently used in Phaecoemulsification cataract surgery and assess the outcome of these substances on the intraocular pressure (IOP), corneal thickness, endothelial cell counts, corneal clarity and initial visual acuity.

Patients and methods: This is a prospective, randomized case series study to compare between two kinds of OVDs used currently in Phaecoemulsification cataract surgery on 40 patients with age range from 55-65 years in the period from June 2009-June 2010, at Al-Jumhory Teaching Hospital, Mosul. Standard procedure was implemented with intraocular lens implantation (IOL). Patients were followed up for three months with particular attention to the first six hours to one day to assess the outcome of surgery in terms of intraocular pressure (IOP), corneal thickness, endothelial cell counts, corneal clarity and visual acuity.

Results: The study enrolled 46 patients, and 40 patients completed the study. There was improvement of visual acuity in all patients. No significant difference in the IOP between the two groups in the first 6 hours to 1 day post operatively (17.90 \pm 2.53$)(18.60 \pm 2.21),(P=0.26)$ and $(17.90 \pm 2.36)(18.55 \pm 2.06),(P=0.941)$ for Appavisc and DisCoVisc related groups respectively. Also there was no difference with regard to both corneal thickness per-operatively and on day one post-operatively and endothelial cell counts $(576.5 \pm 44.04)$

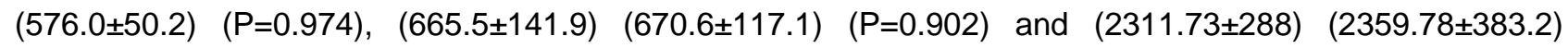
( $P=0.657),(2021.2 \pm 201)(2035.05 \pm 377)(P=0.886)$ for Appavisc and DisCoVisc related groups respectively. In Appavisc related group; four patients had posterior capsular rupture (PCR) and two patients develop grade III corneal edema. In DisCoVisc related group; two patients had PCR and no significant corneal edema. A formal dry vitrectomy done for those developing PCR with successful IOL implantation, and for those developing corneal edema; a frequent instillation of topical corticosteroid was prescribed with improvement of their vision after two weeks post operatively. Initial visual acuity was better for DisCoVisc related group ( $0.74 \pm 0.21)$ than for Appavisc related group (0.52 \pm 0.29$),(P=0.01)$.

Conclusion: Both viscoelastics used had no significant effect on the IOP in the first six hours and first postoperative day, as well as pachymetry and endothelial cell counts both preoperatively and on day one postoperatively, however DisCoVisc was superior to Appavisc regarding corneal clarity and hence visual acuity.
\end{abstract}

\footnotetext{
الخلاصة الهيف من الدراسة: لمقارنة نوعين من السو ائل الثقيلة (DisCoVisc and Appavisc) وملاحظة مدى نأثير هما على ارتفاع

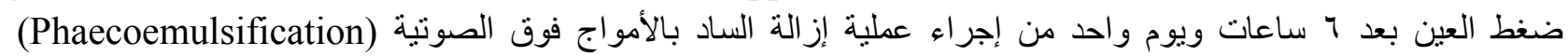
وملاحظة تأثير هذه المو اد على صفاء القرنية وحدة الأبصار. التصميم: در اسة مقطعية.

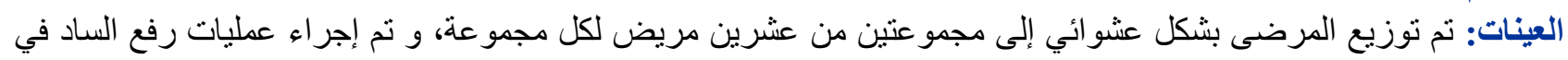

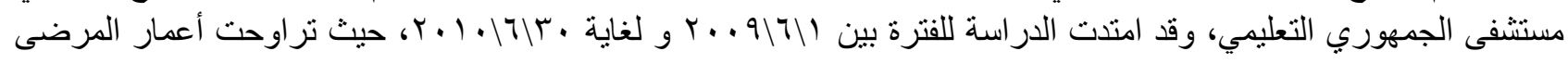




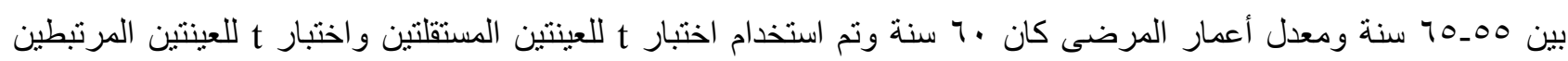

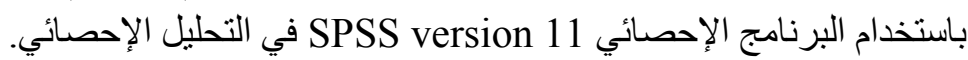

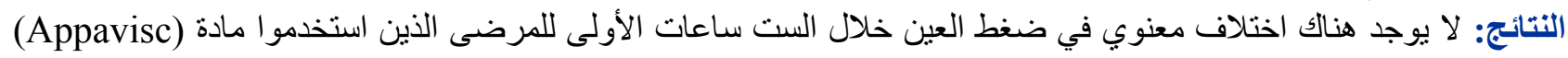

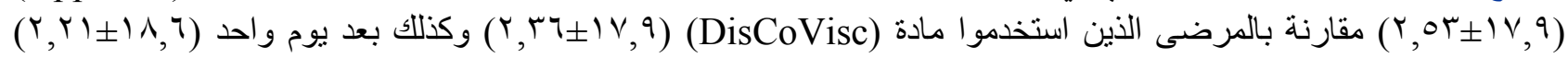

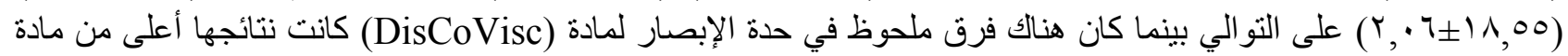

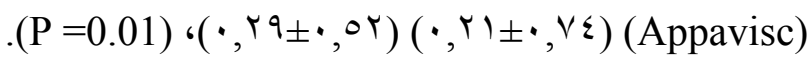

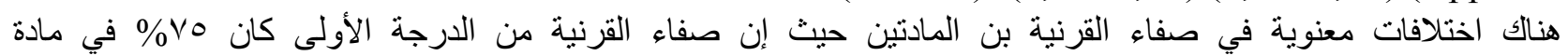
(DisCoVisc)

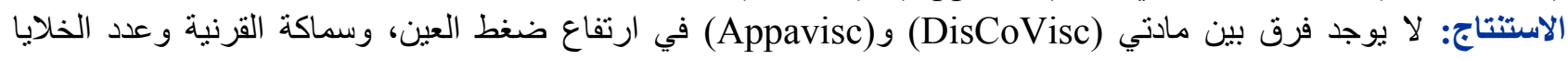

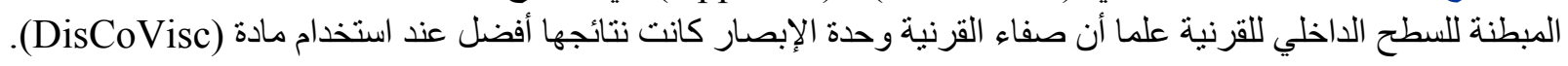

C ataract surgery is a technique described since recorded history, yet it has greatly evolved only in the latter half of the past century ${ }^{(1)}$. The development of intraocular lens and Phaecoemulsification as a technique for cataract removal could be considered as the most significant strides that have been made in this surgical field ${ }^{(1)}$.

Since early 1980s, ophthalmic viscosurgical devices (OVDs) have become invaluable to cataract surgeons for their ability to create space, stabilize tissue and protect endothelial cells, among other attributes ${ }^{(2)}$.

In 1990, there were a limited number of OVDs available, most based on hyaluronic acid, and all could be classified into two groups: higher viscosity cohesive and lower viscosity dispersives ${ }^{(1,2)}$.

Both the capsulorrhexis and intraocular lens (IOL) implantation steps were best achieved with higher viscosity cohesive OVDs, which maximize working space and stability of surgical environment ${ }^{(2,3)}$.

Cohesive or dispersive nature of OVDs is an independent determinant of its behavior ${ }^{(1,2)}$. However, prior to the development of the first viscodispersive OVD, disco-dispersive OVD (DisCoVisc, 4\% sodium chondrotin sulfate/ 1.7\% sodium hyaluronate, Alcon, Fort Worth, Taxes), the correlation between cohesive and zero shear viscosity was very high among ophthalmic cohesive OVDs, because, except for the very low viscosity hydroxypropyle methylcellulose products, the rest were made of long chains of the same molecule, hyaluronic acid ${ }^{(4,5)}$. Consequently, there was no practical sense in having a classification based on both viscosity and cohesion, so a simple rank order of zero-shear viscosity was adequate in early 1990s. Higher viscosities cohesive create space in the anterior chamber and induce a sustained pressure ${ }^{(1)}$.

High viscosity at low shear rates is imperative in OVDs (2). Lower viscosity dispersive offer prolonged retention in the anterior chamber and the ability to partition space ${ }^{(5,6)}$. A well-known example of this type of OVD is Viscot $4 \%$ chondrotin sulfate $13 \%$ soduim hyaluronate, Alcon $^{(2)}$. The viscous dispersive, DisCoVisc, is the unique results of attempting to create an OVD in which zero-shear viscosity and cohesion have been dissociated and independently adjusted, combining the attributes of the two OVD groups, so it has the desired viscosity of Helon, with dispersion characteristics similar to Viscot ${ }^{(7)}$.

Two of the most feared potential complications surgeons face during Phaecoemulsification are the creation of a hole in the posterior capsule and damaging the cornea ${ }^{(8)}$. During phaco, there are only two major roles that OVD most play: It must stabilize the anterior chamber and protect corneal endothelium ${ }^{(8)}$. Within reasonable limits, the deeper the anterior chamber, the better during surgery ${ }^{(9)}$. Historically, surgeons were forced to pick between a deep, stable chamber and high retention of the OVD, because we accepted that higher retention equated with lower viscosity ${ }^{(10)}$.

Ideally, we use two OVDs in the soft shell technique ${ }^{(4)}$. DisCoVisc combines the two main attributes of high zero-shear viscosity and prolonged retention due to dispersive nature into one OVD ${ }^{(11,12)}$. Surgery is simpler because there is no need for $2^{\text {nd }}$ OVD injection ${ }^{(10)}$. DisCoVisc, by combining higher viscosity and dispersion into a 
single OVD, has approached the dilemma of OVD choice differently than Helon 5, which is an extremely high viscosity OVD that becomes pseudo-dispersive when exposed to high stress ${ }^{(13)}$.

Chondrotin sulfate, the major chemical component, other than hyaluronic acid, commonly obtained from shark cartilage, in Viscot and DisCoVisc is very dispersive with a low viscosity, and remains adjacent to the corneal endothelium for longer than sodium hyaluronate ${ }^{(11,14)}$. Chondrotin sulfate works well when it is mixed with sodium hyaluronate than it dose on it is own because it achieves a much better viscosity profile ${ }^{(8,9)}$. This account for the superior behavior of Viscot and DisCoVisc in cataract surgery, compared to chondrotin sulfate alone ${ }^{(7)}$. The enhanced retention of both Viscot and DisCoVisc during Phaecoemulsification is what confers their superior endothelial protective properties upon them ${ }^{(13)}$.

Hydroxypropyle methylcellulose (HPMC) is another viscoelastic substance that dose not occurs naturally in animal tissues but is widely distributed in plant fibers like cotton and wood. The structure of the commercial product is a cellulose polymer to increase the hydrophilic propensity of the material. Methylcellulose is a non-physiologic compound that doesn't appear to be metabolized intraocularly. It is eventually eliminated in the aqueous but can be easily irrigated from the eye; Appavisc is an example of this group ${ }^{(13)}$.

Choosing DisCoVisc OVD during Phaecoemulsification procedure may allow for protection of endothelial cells while suppressing the formation of free radicals. This may be the reason for the superior protective effect on the corneal endothelial cells of DisCoVisc compared with other OVDs ${ }^{(13)}$.

The aim of this study is to compare two types of viscoelastic substances (DisCoVisc and Appavisc) that are used currently in Phaecoemulsification cataract surgery and their effects on the IOP, corneal pachymetry, endothelial cell counts, corneal clarity and hence the visual acuity post operatively.

\section{PATIENTS AND METHODS}

Forty-six patients were enrolled in this study which was performed at Al-Jumhory Teaching Hospital, Mosul, in the period from 1/6/2009 to 30/06/2010. Four patients were excluded from the study (two because of other coexisting ocular pathology and two because another viscoelastic substance was used accidentally) and two patients lost for followup.

All patients were complaining from reduction of visual acuity that interferes with their daily activities and/or spoil their life quality, they were fully assessed by the ophthalmologist (author) and proved to have cataract (grade III and IV) that interfere with their daily activities. The age was ranging from 55-65 years with a mean of 60 years; there were 33 female and 7 male patients.

Pre-operative evaluation of the patients included the measurement of visual acuity, refraction, an examination of anterior segment of the eye under slit lamp biomicroscope, central corneal thickness and fundus evaluation through a dilated pupil. Intraocular pressure was recorded pre-operatively with a Goldmann applination tonometer in all patients. Keratometry was performed, while the axial length was measured using an $A$ scan biometer. The power of intraocular lens was calculated in all patients using SRK (Sanders, Retzlaff, Kraff) formula. History of any systemic illness was excluded and blood pressure was measured on admission.

\section{Surgical technique}

Surgeries were performed under local anesthesia using $1 \%$ xylocain. Pupillary dilatation was achieved by a combination of topical tropicamide $1 \%$ and $2.5 \%$ phenylephrine. A $3.2 \mathrm{~mm}$ clear corneal tunnel was created either superiorly or temporally depending upon the steeper axis; side port entry was made with the help of microvitreoretinal (MVR) blade. OVD was injected into the anterior chamber according to the randomization, and capsulorrhexis was performed using Uttrata capsulorrhexis forceps. Hydrodissection and hydrodelination were performed to achieve free rotation of nucleus. Phaecoemulsification was done using horizontal chopping technique by Nidek CV-7000 (Nidek Co, Ltd, Japan) which was followed by a thorough irrigation and aspiration of cortical matter. Capsular bag was inflated with viscoelastic and implantation of a single piece foldable acrylic lens using an injector system. \{(ACRYFOLD ${ }^{\circ}$, HEMA, UV absorbing optic implant was used for all patients, this product marketed by Appassamy associates, India in collaboration with GANTEC 
CORPORATION (U.S.A) 2354 Hassell Road, Suite D, Hoffman Estate, IL 60195. The optic diameter of the implant was $6.00 \mathrm{~mm}$ squared edge all over, length $12.5 \mathrm{~mm}$ and A-constant 118 . This product is a single piece biconvex foldable IOL comes with a package that also include one delivery system that include one cartridge and soft tipped injector\} was implanted in all the patients. The OVD was completely aspired out with the rock and roll technique. The irrigating solution and Phaecoemulsification machine were similar in all the two groups.

The intra-operative parameters recorded were the type of OVD, Mean Phaco time (min): (2.1 \pm 1.0$)$, effective phaco time (sec): $(36.1 \pm 20.4)$, mean total surgical time $(\mathrm{min}):(16.5 \pm 3.6)$ and OVS removal time (sec): $(55.47 \pm 6.6)$ for both groups.

The Phaecoemulsification parameters were as follow: ultrasound (us) $70-90 \%$, vacuum $180-200$ $\mathrm{mmHg}$, flow rate $30-33 \mathrm{cc} /$ minute, with bottle height $65 \mathrm{~cm}$.

Post-operatively, all patients were prescribed Prednisolone acetate 1\% and Ciprofloxacin 0.3\% QID each for four weeks.

\section{RESULTS}

Forty patients underwent Phaecoemulsification cataract surgery for reduction of their visual acuity that interferes with their daily activities. No major complications, namely suprachoroidal hemorrhage, retinal detachment, wound burn or iris injury at time of surgery.

The follow up period extends from the same operative day up to three months and then the follow up discontinued. It seems from the current situation that there is no difference in the intraocular pressure measurement in the first six hours between the two groups $(17.9 \pm 2.53)$ for Appavisc, $(17.9 \pm 2.36$ ) for DisCoVisc $=1.00$ (Table $1 \& 2$ ) (Figure 1). And in the first postoperative day $(18.6 \pm 2.21)$ for Appavisc, $(18.55 \pm 2.06)$ for DisCoVisc $=0.94100$ (Table 1) (Figure 2).

Four patients from Appavisc related group and only two patients from DisCoVisc related group develop posterior capsular rupture (PCR), these related probably to under sized capsulorrhexis, damage of the anterior capsule by the second instrument (Agarwals' chopper), aggressive Phaecoemulsification and/or aggressive phaco settings, however all cases of PCR dealt with intraoperatively by doing dry vitrectomy by inserting vitrectomy probe through one side port and Viscocannula through other side port to maintain anterior chamber stability. Only one patient from Appavisc related group develop mild wound leakage that observed under the slit-lamp examination with the adjuvant use of Fluorescein sterile paper strip (Seidel test), a soft bandage contact lens was inserted in the first six hours in the private clinic, fortunately this problem resolved on the next day. Two patients from Appavisc related group develop grade III corneal edema (Table 3), this problem attributed to the so called stony hard nature of the lens in these two cases together with the aggressive phaco setting and the use of woodcutter technique rather than ordinary horizontal chopping. With the frequent instillation of topical Prednisolone acetate 1\% hourly, the edema resolved in a couple of week. Mild postoperative iritis (Grade I-II) developed in most patients of both groups, which was halted by postoperative instillation of topical Prednisolone acetate 1\% for two weeks. No anterior chamber surge phenomenon happened because phaco hand piece had been checked in the priming chamber filled with balanced salt solution (BSS) fluid prior to insertion of phaco hand piece in the anterior chamber, further checking that no tube kinking nor air bubbles in the whole tubing system together with a proper main and side port incision construction that prevent fluid leaking.

It was evidenced by using Snellen chart for testing visual acuity and slit-lamp biomicroscope examination for assessment corneal clarity that DisCoVisc achieves better results than Appavisc in terms of visual acuity $(0.52 \pm 0.29)$ for Appavisc related group, $(0.74 \pm 0.21)$ for DisCoVisc related group ( $P=0.01$ ), (Table 1 and 2) (Figure 3).

Table 1. IOP and initial visual acuity results for Appavisc and DisCoVisc groups.

\begin{tabular}{|c|c|c|c|}
\hline \multirow{2}{*}{ Parameter } & $\begin{array}{c}\text { Appavisc } \\
(n=20)\end{array}$ & $\begin{array}{c}\text { DisCoVisc } \\
(n=20)\end{array}$ & \multirow{2}{*}{$\begin{array}{c}P^{*} \text { - } \\
\text { Value }\end{array}$} \\
\hline & $\begin{array}{c}\text { Mean } \pm \\
\text { SD }\end{array}$ & $\begin{array}{l}\text { Mean } \pm \\
\text { SD }\end{array}$ & \\
\hline $\begin{array}{l}\text { IOP (6-hours) } \\
\text { Post operative }\end{array}$ & $\begin{array}{c}17.90 \pm \\
2.53\end{array}$ & $\begin{array}{c}17.90 \pm \\
2.36\end{array}$ & 1.000 \\
\hline $\begin{array}{l}\text { IOP (1-Day) Post } \\
\text { operative }\end{array}$ & $\begin{array}{c}18.60 \pm \\
2.21\end{array}$ & $\begin{array}{c}18.55 \pm \\
2.06\end{array}$ & 0.941 \\
\hline $\begin{array}{l}\text { Initial Visual } \\
\text { Acuity }\end{array}$ & $\begin{array}{l}0.52 \pm \\
0.29\end{array}$ & $0.74 \pm 0.21$ & 0.010 \\
\hline
\end{tabular}

* Unpaired t- test was used ${ }^{(15)}$ 
There was no significant increase in central corneal thickness between the two groups in the immediate postoperative period and also at the end of three months following surgery. Also there was no significant difference in the change in endothelial count in eyes between the two groups, (Table 4).

Table 2. IOP measurements in the first six hours and first post-operative day in Appavisc and DisCoVisc related groups.

\begin{tabular}{|l|c|c|c|}
\hline \multirow{3}{*}{ Parameter } & $\begin{array}{c}\text { After 6- } \\
\text { hours } \\
(\mathbf{n = 2 0})\end{array}$ & $\begin{array}{c}\text { After 1- } \\
\text { Day }(\mathbf{n = 2 0})\end{array}$ & \multirow{2}{*}{$\begin{array}{c}\text { P*- } \\
\text { Value }\end{array}$} \\
\cline { 2 - 3 } & Mean \pm SD & Mean \pm SD & \\
\hline Appavisc & $\begin{array}{c}17.90 \pm \\
2.53\end{array}$ & $\begin{array}{c}18.60 \pm \\
2.21\end{array}$ & \multirow{2}{*}{0.286} \\
\hline DisCoVisc & $\begin{array}{c}17.90 \pm \\
2.36\end{array}$ & $\begin{array}{c}18.55 \pm \\
2.06\end{array}$ & 0.941 \\
\hline
\end{tabular}

* Paired t- test was used ${ }^{(15)}$

Table 3. Grading of corneal clarity.

\begin{tabular}{|c|c|c|c|}
\hline $\begin{array}{c}\text { Grading of } \\
\text { corneal } \\
\text { clarity }\end{array}$ & DisCoVisc & Appavisc & \multirow{2}{*}{ P $^{*}$-value } \\
\cline { 2 - 3 } & No. (\%) & No. (\%) & \\
\hline Grade I & $15(75.0)$ & $6(30.0)$ & 0.010 \\
Grade II & $3(15.0)$ & $10(50.0)$ & 0.041 \\
Grade III & $2(10.0)$ & $4(20.0)$ & 0.661 \\
Grade IV & ---- & ---- & ------ \\
\hline Total & $20(100 \%)$ & $20(100 \%)$ & $P^{* \star}=$ \\
& & & 0.016 \\
\hline
\end{tabular}

${ }^{*}$ Fisher's exact test ${ }^{(15)} .{ }^{*} X^{2}$-test was used (8.293) d. $\mathrm{f}=2$.

${ }^{* * *}$ Grading of corneal clarity

Grade I: crystal clear cornea.

Grade II: hazy cornea but with visible iris details.

Grade III: stromal haze obscuring iris details.

Grade IV: opaque cornea.

Table 4. Pre-operative and post-operative corneal characteristics for the patients.

\begin{tabular}{|l|c|c|c|}
\hline \multicolumn{1}{|c|}{ Parameter } & $\begin{array}{c}\text { Appavisc } \\
(\mathbf{n = 2 0})\end{array}$ & $\begin{array}{c}\text { DisCoVisc } \\
(\mathbf{n = 2 0 )}\end{array}$ & $\begin{array}{c}\mathbf{P}^{*} \text { - } \\
\text { value }\end{array}$ \\
\hline $\begin{array}{l}\text { Corneal } \\
\text { thickness in }\end{array}$ & & & \\
micron $(\mu \mathrm{m})$ & & & \\
Pre-operative & $567.5 \pm 44.07$ & $576.0 \pm 50.2$ & 0.974 \\
Day one & $665.5 \pm 141.9$ & $670.6 \pm 117.1$ & 0.902 \\
\hline Endothelial & & & \\
cell count & & & \\
(cells/ $\left.\mathrm{mm}^{2}\right)$ & & & \\
Pre-operative & $2311.73 \pm 288.0$ & $2359.78 \pm 383.2$ & 0.657 \\
Day one & $2021.20 \pm 201.0$ & $2035.05 \pm 377.0$ & 0.886 \\
\hline
\end{tabular}

*Unpaired t- test was used ${ }^{(15)}$.

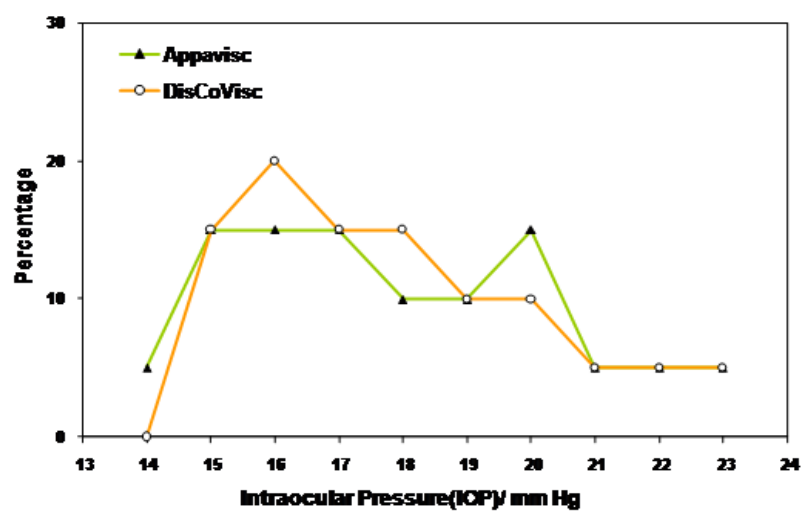

Figure 1. Intraocular Pressure (IOP) (6-Hours) Postoperatively.

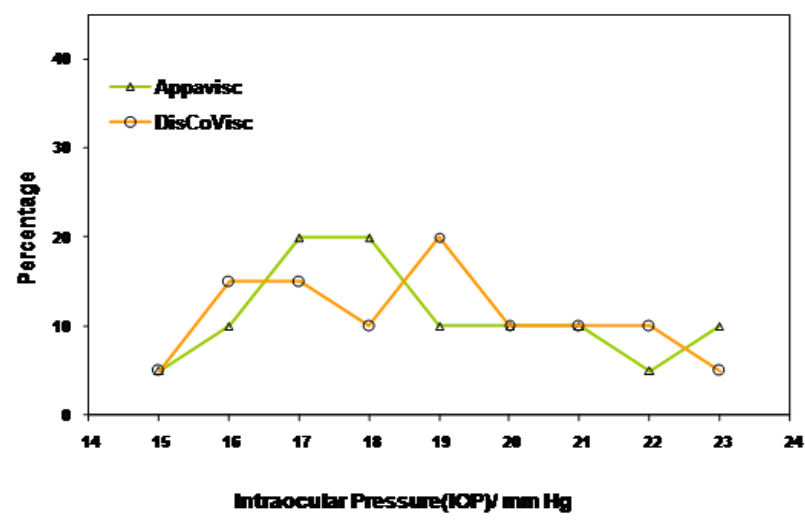

Figure 2. Intraocular Pressure (IOP) (1-Day) Postoperatively.

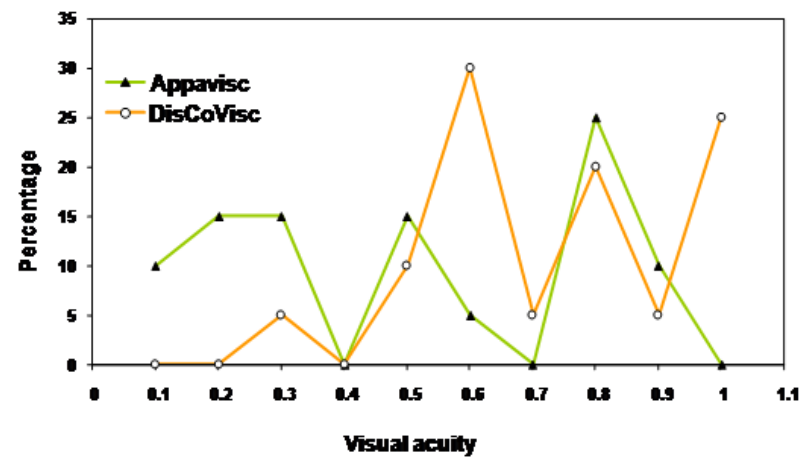

Figure 3. Initial visual acuity (1-Day) Post Operatively.

\section{DISCUSSION}

Surveys over the last two years demonstrate that $95 \%$ of surgeons believe that endothelial protection is their key factors, but there are a number of other attributes with viscoelastics that are also important ${ }^{(14)}$.

The most important criterion in evaluating the quality of surgery is the visual acuity in the early post-operative period and that translate to corneal 
clarity ${ }^{(6)}$. When one chooses OVD, we want to choose something that will make our surgery as easy as we possibly can make it for the patient, and then we want to have a clear corneas and nice outcomes the first day ${ }^{(9,12)}$.

It is obviously most pleasant for us to walk on the first post-operative visit and the patient sees $20 / 20$, the cornea is clear, and we have no headache ${ }^{(10,12)}$. We want to achieve clear corneas, which we all agree and everyone has agreed for years-that chondrotin sulfate is the best content of a device to preserve good, healthy, clear, corneas on day one, but in many places we want to save money that is the issue $e^{(6,12)}$.

When we take a look at the global cost to the cataract patient, it is not just how much the product itself costs ${ }^{(1,13)}$. It is about what we have to do to take care of that patient through the peri-operative period, the number of visits to the office, perhaps the number of eye drops necessary to quiet the inflamed eye. The global cost of surgery in the entire peri-operative period is the key determinant that needs to be considered versus the initial cost of just the product at the time of the procedure ${ }^{(6)}$. The patient pays once because that is high percentage of situations in many markets now that Phaecoemulsification is expanding. Patient does not understand the effect of a good versus poor OVD on their long-term care ${ }^{(5,6)}$.

This can be terribly significant if the cornea were to be damaged when such a simple change can help prevent that issue. A small cost upgrade would save them overtime ${ }^{(6)}$.

Intraocular pressure rise is one of the most interesting, misunderstood problems in OVDs. A post-operative IOP increase following uncomplicated Phaecoemulsification may be related to the amount of retained viscoelastic agent at the end of surgery ${ }^{(14)}$. Residual viscoelastics mechanically obstruct the trabecular outflow pathway, decreasing the outflow facility (11). Different responses from various viscoelastic agents are explained by difference in their biophysical properties ${ }^{(10)}$. The clearance of viscoelastic agent through the trabecular meshwork is dependent upon the viscosity, molecular weight, and molecular chain length of the materials ${ }^{(14)}$. Therefore; the higher the viscosity and the molecular weight of the viscoelastic agent, the slower clearance through the trabecular meshwork, resulting in a greater risk of elevated IOP ${ }^{(13,14)}$. The problem is the IOP rise maximal from $6-12$ hours post-operatively ${ }^{(13)}$. No one measure pressure six or twelve hours postoperatively. No one is going to bring patient back at 4:00 $\mathrm{AM}$ or mid-night to measure their pressure $^{(12)}$.

If left in the eye, all OVDs will cause IOP spikes, and the pressure spike is not so high on the first day post-operatively, because we missed it at 6-10 hours post-operatively ${ }^{(13)}$.

Intraocular pressure in eyes that we see on first post-operative day is directly related to the amount of inflammation ${ }^{(7)}$. If we get an inflamed eye, the IOP can be very high ${ }^{(7)}$. The other point is that we spend a lot of time trying to remove OVD, and when we do that, we are going to use much more infusion fluid, which is also damaging to the intraocular structures ${ }^{(8,10)}$. Surgeons are limited in how they perform by their complication rate. If one have high complication rate as a consequence of using inferior devices we are really destroying our own practice ${ }^{(8)}$. Surgeons want to use good OVDs to make their cases easier because it makes everything much better. It lowers their complication rate ${ }^{(14)}$.

Really no difference in the IOP measurement in the first six hours and first post operative day (Table 1 and 2). But the corneal clarity and hence the initial visual acuity was better with the $2^{\text {nd }}$ group (Table 3) (Figure 3). It seems also that the complication rates were much less and surgery runs more smoothly with the $2^{\text {nd }}$ group. Posterior capsular tear $n=4$ and corneal edema $n=2$ post operatively in Appavisc-treated group, mean while posterior capsular tear $n=2$, and no corneal edema at all in DisCoVisc-treated group provided that we use the same phaco parameters in both groups.

Fortunately, both products now currently available in the market, the prices subjected to a regional variations, for DisCoVisc OVD its prices (including the taxes) ranged between 25-30 U. S dollars which comes as a sterile, non-pyrogenic single use, ophthalmic viscosurgical devices supplied in a disposable syringe delivering $0.5 \mathrm{ml}$ with a 27 gauge cannula and cannula locking ring, packaged in a blister tray. The product (viscosurgical device solution is aseptically processed and the syringe exterior is sterilized by ethylene oxide. On other hand, the price of 
Appavisc OVD (taxes included) ranged between 10-12 U.S dollars which comes as a preservative free, sterile solution supplied in a disposable syringe delivering $2 \mathrm{ml}$ with a 27 gauge cannula, packaged in a nylon pouch with steam sterilization, the syringe exterior is sterilized by ethylene oxide.

\section{CONCLUSION}

DisCoVisc is superior to Appavisc in Phaecoemulsification with regards to the corneal clarity and visual acuity, provided that both products had no significant effect on the IOP in the first six hours to one day post operatively. Also there is no significant difference between the two groups with regard of corneal pachymetry and endothelial cell count both at pre-operative day and day one post-operatively. DisCoVisc can be used successfully in all steps of Phaecoemulsification cataract surgery. DisCoVisc is the first OVD that combine high viscosity and dispersion.

\section{REFRENCES}

1. Arshinoff SA, Jafari M. New classification of ophthalmic viscosurgical devices-2005. J Cataract Refract Surg. 2005; 31:2167-2171.

2. Bissen-Miyajima $\mathrm{H}$. In vitro behavior of ophthalmic viscosurgical devices during Phaecoemulsification. J Cataract Refract Surg. 2006; 32:1026-1031.

3. Oshika T, Okamoto F, Kaji Y, et al. Retention and removal of a new viscous dispersive ophthalmic viscosurgical device during cataract surgery in animal eyes. Br J Ophthalmol. 2006; 90:458-7.

4. Arshinoff SA. Dispersive-cohesive visoelastics soft shell technique. J Cataract Refract Surg. 1999; 25:167-73.

5. Arshinoff SA. Dispersive and cohesive viscoelastic materials in Phaecoemulsific- ation. Ophthalmic Pract 1995; 13:98-104.

6. Oshika T, Eguchi S, Oki K, et al. Clinical comparison of Healon 5 and Healon in phacoemulsification and intraocular lens implantation: randomized multicenter study. J Cataract Refract Surg 2004; 30(2):357-362.
7. Vajpayee RB, Verma K, Sinha R, et al. Comparative evaluation of efficacy and safety of ophthalmic viscosurgical devices in phacoemulsification. BMC ophthalmol 2005;5:17-22.

8. Lindstorm RL. Foldable Intraocular Lenses. In: Steinert RF, ed. Cararact Surgery: Technique, Complications, and Management. Philadelphia: WB Saunders; 1999. p.279-294.

9. Rainer G, Menapace R, Findl O, et al. Effect of a fixed dorzolamide-timolol combination on intraocular pressure after small-incision cataract surgery with Viscot. J Cataract Refract Surg 2003; 29(9):174852.

10. Kiss B, Findl O, Menapace R, et al. Corneal endothelial cell protection with a dispersive viscoelastic material and an irrigation solution during Phaecoemulsifi- cation: low-cost versus expensive combination. J Cataract Refrac Surg 2003; 29(4): 733-40.

11. Rainer G, Menapace R, Findl O, et al. Intraocular pressure rise after small incision cataract surgery: a randomized intraindividual comparision of two dispersive viscoelastic agents. $\mathrm{Br} \mathrm{J}$ Ophthalmol 2001; 85(2):139-42.

12. Lane SS, Lindstorm RL. Viscoelastic agents: formulation, clinical applications, and complications. In: Steinert RF, Fine IH, eds. Cataract Surgery: Technique, Complications, \& Management. Philadelphia: WB Saunders; 1995:37-45.

13. Liesegang TJ. Viscoelastic substances in ophthalmology. Surv Ophthalmol. 1990; 34: 268293.

14. Rainer G, Menapace R, Findl O, Georgopoulos M, Kiss B, Petternel V. Intraocular pressure after small incision cataract surgery with Healon5 and Viscot. J Cataract Refract Surg. 2000; 26:271-276.

15. Chap T. Introductory Biostatistics. International edition. USA: John Wiley and Sons; 2003. p.224256. 